\title{
Synthesis and pharmacological evaluation of novel epidermal growth factor receptor inhibitors against prostate tumor cells
}

\author{
YI ZHI $^{1,2}$, XIAOJUN WU ${ }^{1}$, WENHAO SHEN ${ }^{1}$, YONGQUAN WANG ${ }^{1}$, XIAOZHOU ZHOU $^{1}$, \\ PENG HE ${ }^{1}$, JINHONG PAN $^{1}$, ZHIWEN CHEN ${ }^{1}$, WEIBING LI $^{2}$ and ZHANSONG ZHOU ${ }^{1}$ \\ ${ }^{1}$ Urology Institute of People Liberation Army, Southwest Hospital, Third Military Medical University, Chongqing 400038; \\ ${ }^{2}$ Department of Urology, Third Affiliated Hospital of Chongqing Medical University, Chongqing 401120, P.R. China
}

Received June 24, 2016; Accepted August 15, 2017

DOI: $10.3892 / \mathrm{ol} .2018 .9438$

\begin{abstract}
The aim of the present study was to investigate the activities of novel synthetic epidermal growth factor receptor (EGFR) inhibitors (ZINC05463076, ZINC2102846 and ZINC19901103) against prostate tumors, in vitro models and investigate the potential underlying mechanisms. A panel of prostate tumor cell lines (LNCaP, DU-145, PC-3 and LNCaP-AI cells) were used to evaluate antitumor activity of ZINC05463076, ZINC2102846, and ZINC19901103 in vitro. Cell growth and clonal formation were determined by MTT assay and Soft agar colony formation assay, respectively. An EGFR kinase assay following treatment of the compounds was performed by ELISA. Cell cycle-regulating proteins, including cyclin-dependent kinase (CDK)1, CKD2, CKD4 and inhibitory effects of these compounds on downstream signaling were analyzed by western blotting. Flow cytometry was performed to investigate apoptosis and cell cycle phases of the treated cells. It was revealed that all compounds synthesized in the present study demonstrated significant EGFR inhibition abilities, compared with approved EGFR inhibitor drug gefitinib. Treatment of LNCaP, DU-145, PC3 and LNCaP-AI cells with these compounds revealed cell proliferation inhibition and colony formation suppression dose-dependently in vitro. The agents impaired phosphorylation of EGFR and extracellular signal-regulated kinase 1/2 and suppressed their downstream signaling. In addition, these novel synthetic agents decreased the expression level
\end{abstract}

Correspondence to: Dr Zhansong Zhou, Urology Institute of People Liberation Army, Southwest Hospital, Third Military Medical University, 30 Gaotanyan Street, Shapingba, Chongqing 400038, P.R. China

E-mail: zhouzhansong@sohu.com

Dr Weibing Li, Department of Urology, Third Affiliated Hospital of Chongqing Medical University, 1 Shuanglong Street, Yubei, Chongqing 401120, P.R. China

E-mail: liweibing63@aliyun.com

Key words: epidermal growth factor receptor, prostate cancer, anticancer agent, tyrosine kinase, cell cycle of survivin, which may induce $\mathrm{G}_{1}$ cell cycle phase arrest and cell apoptosis in PCa cells subsequently. Collectively, ZINC05463076, ZINC2102846 and ZINC19901103 exhibited significant antitumor activity in human prostate tumors in vitro, by inhibiting EGFR and promoting apoptosis, which suggested a rationale for clinical development in prostate tumor therapy.

\section{Introduction}

Prostate cancer (PCa) is one of the most common types of cancer and is the second leading cause of cancer-associated mortality in males in the USA (1). The majority of prostate tumor cells remain androgen-sensitive initially, as the cancer cells develop from the normal epithelium (2). At a later stage, these tumor prostate cancer cells progress to the aggressive androgen-independent state with increased invasion capacity and proliferation, in a number of cases (3). Chemotherapy, as the first-line treatment, has been used for treatment of patients with androgen-independent $\mathrm{PCa}$ in combination with docetaxel; however, the median survival time of these patients is only approximately 18 months (4). Therefore, new approaches for treating PCa with high efficiency and safety are required.

Previous findings suggest the proliferation of androgen-independent $\mathrm{PCa}$ is mediated by autocrine factors (5). For example, epidermal growth factor receptor (EGFR)-mediated signaling pathways have been suggested to play a role in the progress of androgen-independent $\mathrm{PCa}$, which may interact with the androgen receptor (AR) directly in the absence of ligands and promote tumor cell growth, the ability of invasion and metastasis by activating the downstream signaling pathways (6). Previous findings also detected that EGFR is overexpressed in the majority of PCa cells and its expression level was revealed to be significantly associated with progression from an androgen-dependent tumor to an androgen-independent tumor; conversely, interruption of the EGFR-mediated signaling pathway promotes apoptosis and inhibits proliferation in these tumor cells (7). Accordingly, targeting EGFR may serve as a potential approach to treat androgen-independent $\mathrm{PCa}$ (8).

Since the discovery of protein kinase activity in 1954, multiple kinase inhibitors have been identified in various 
studies, which have been widely used in clinical practice $(9,10)$. Accordingly, high-throughput docking has been considered to be the key entrance for drug discovery since 2000, which may forecast an optimized conformation for the protein and ligand molecule $(11,12)$. The present study investigated three potential drugs of ZINC05463076, ZINC2102846 and ZINC19901103 by docking simulation based on known protein kinase inhibitors, and explored their therapeutic effects on PCa (13).

The aim of the present study was to identify whether ZINC05463076, ZINC2102846 and ZINC19901103, three potent and specific antagonists of EGFR, exerted antineoplastic effects on PCa cell lines (LNCaP, PC-3, DU-145 and LNCaP-AI) and the potential underlying mechanisms. The findings revealed that the three compounds exerted anti-proliferative effects on PCa cell lines and promoted tumor suppression via $G_{0} / G_{1}$ cell cycle arrest, and further inhibited tumor cell migration and invasion. The present study investigated the effects of these compounds on the expression levels of members of the inhibitor of apoptosis (IAP) family, which is associated with anti-apoptotic and pro-apoptotic proteins, including survivin, cyclin-dependent kinase (CDK)1, CDK2 and CDK6 in pancreatic cancer cell lines by western blotting. The results of the present study revealed that the blockade of EGFR by ZINC05463076, ZINC2102846 and ZINC19901103 may be a potential targeted therapeutic strategy against $\mathrm{PCa}$.

\section{Materials and methods}

Protein and ligand database preparation. A structure-based virtual screening was performed to identify potential EGFR kinase inhibitors. The docking-ready 3D coordinates of the X-ray structure of EGFR kinase (PDB: 1XKK) and its cognate ligand were downloaded from the scPDB (http://bioinfo-pharma.u-strasbg.fr/scPDB). The present study selected the X-ray structure $1 \mathrm{XKK}$ as it had been successfully used in previous structure-based virtual screenings $(14,15)$. A commercially available compound database from the Specs company (http://www.specs.net) was selected to screen against EGFR kinase. The docking-ready file of the Specs database was downloaded from the ZINC database (http://zinc.docking.org), which is a free database of commercially available compounds for virtual screening.

Docking computation. Surflex-Dock, a docking software, implemented in SYBYL-X (version 1.3; http://www.tripos. com) was used to perform virtual screening. Surflex-Dock uses a protocol file to describe the profile of a binding site. In the present study, the bound-ligand of $1 \mathrm{XKK}$ was used to guide the protocol generation in the default mode. Subsequently, a flexible ligand docking virtual screening was performed using Surflex-Dock in the default mode with the groom option. In order to evaluate the docking accuracy, a self-docking experiment was performed using the computed 3D structure of cognate ligand.

Virtual screening. The top pose of each molecule was analyzed by virtual screening. The root-mean-square-deviation (RMSD) between the top pose and the bound-ligand was used to measure of the difference between values predicted by docking computation (16). A RMSD of $<2 \AA$ is usually considered an upper limit of drug discovery (17). The docked molecules were ranked in descending order by docking score (the greater the score, the more potential activity is). The present study focused on the molecules with a significantly good score. In order to evaluate the significance of the docking score, the present study determined a P-value by using the inverse cumulative distribution of docking scores of the 15,966 decoy molecules (http://dud.docking.org), which have been used to evaluate the performance of docking-based virtual screening approaches (18). Sock scoring included a dock score $\geq 10 \mathrm{pKd}$, crash score $\geq-2 \mathrm{pKd}$ and polar score $\geq 2 \mathrm{pKd}$.

Cells and cell culture conditions. LNCaP,PC-3 and DU-145 cell lines were obtained from the Cell Resource Center (Institute of Basic Medical Sciences, Chinese Academy of Sciences, Shanghai, China). The LNCaP, PC-3 and DU-145 cell lines were grown in RPMI-1640 medium (Gibco; Thermo Fisher Scientific, Inc., Waltham, MA, USA) supplemented with $10 \%$ fetal bovine serum (FBS), $20 \mathrm{mM}$ HEPES buffer, $50 \mu \mathrm{g} / \mathrm{ml}$ gentamycin, $0.1 \mathrm{IU} / \mathrm{ml}$ insulin and $5 \mu \mathrm{g} / \mathrm{ml}$ transferrin. The fibroblasts were cultured in Dulbecco's modified Eagle's medium supplemented with $10 \%$ FBS, 2 mmol/L-glutamine and $1 \%$ penicillin-streptomycin.

To establish LNCaP-AI (androgen-independent) cells, a specific subline of LNCaP cells, LNCaP cells were maintained in RPMI-1640 supplemented with androgen-depleted $10 \%$ FBS for 3 months (19). The majority of the cells died in the final 12 weeks. Two of the few survival colonies were transferred to 96-well plates and cultured for an androgen-depleted experiment. The two isolates were assigned as LNCaP-AI no. 1 and 2 cell lines. Western blotting revealed that there was a markedly lower AR expression level in the two isolates, compared with the controls, and cell growth assays also confirmed their androgen independence (data not shown). LNCaP-AI cells were seeded in 16-well microculture plates in FBS-containing RPMI-1640 medium. All the cells in this experiment were plated at a constant density $\left(3 \times 10^{5}\right.$ cells/well in a 6 -well plate) to obtain identical experimental conditions in order to achieve a high accuracy of the various evaluations performed. All cultures were incubated at $37^{\circ} \mathrm{C}$ in a humidified, $95 \%$ air and $5 \% \mathrm{CO}_{2}$ atmosphere. The medium, containing RPMI-1640, $10 \%$ neonatal calf serum, $100 \mathrm{U} / \mathrm{ml}$ of penicillin and $100 \mu \mathrm{g} / \mathrm{ml}$ of streptomycin, was replaced every 2-3 days.

ELISA. The method of ELISA was established to estimate the phosphorylation degree of EGFR. Cells were plated in 6-well flat-bottom plates to full confluence and incubated for $24 \mathrm{~h}$ at $37^{\circ} \mathrm{C}$ in a humidified atmosphere containing $5 \% \mathrm{CO}_{2}$. Supernatants were removed and evaluated using an ELISA kit (cat. no. ab126441; Abcam, Cambridge, MA, USA), according to the manufacturer's protocol. Color development was determined using a microplate reader (MK3; Thermo Fisher Scientific, Inc.) set to $490 \mathrm{~nm}$.

Cytotoxic and anti-proliferative activities. The cell viability was assessed using a standard MTT dye reduction assay, according to the manufacturer's instructions (Sigma-Aldrich; Merck KGaA, Darmstadt, Germany). Accordingly, each 


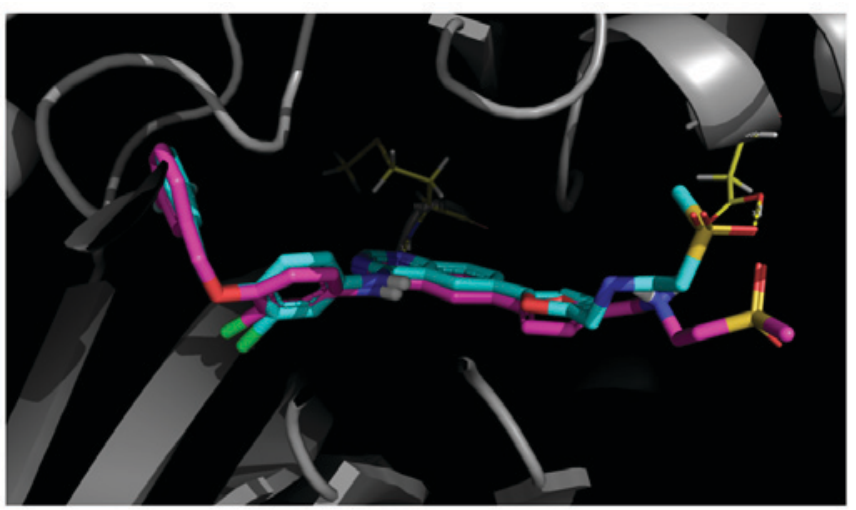

Figure 1. The result of self-docking. Cyan, Co-crystal ligand; magenta, The top pose.

of the three substances, ZINC05463076, ZINC2102846, ZINC19901103, were analyzed at five different concentrations ranging from 0.01 to $100 \mu \mathrm{mol}, 6.25,12.5,25,50$ and $100 \mu$ mol. Briefly, 3x10 $10^{5}$ PC-3, DU-145, LNCaP or LNCaP-AI were plated into each well of 6 -well flat-bottomed microplates and incubated overnight at $37^{\circ} \mathrm{C}$ in $5 \% \mathrm{CO}_{2}$. Subsequently, they were untreated (controls) or treated for $72 \mathrm{~h}$ with each experimental compound at $37^{\circ} \mathrm{C}$. Results were expressed as the mean cell number.

Soft agar colony formation assay. Single cell suspension of LNCap pre-treated cells with ZINC05463076, ZINC2102846 or ZINC19901103 at their half-maximal inhibitory concentrations $\left(\mathrm{IC}_{50} \mathrm{~s}\right), 13.34$ and $13.19 \mu \mathrm{mol} / 1 \mathrm{ZINC} 05463076$ for $\mathrm{LNCaP}$ and LNCaP-AI cells, respectively, for $72 \mathrm{~h}$ at $37^{\circ} \mathrm{C}$ were acquired by passing the cells through a fine needle. A total of 5,000 LNCaP and LNCaP-AI cells following treatment with ZINC05463076, ZINC2102846 or ZINC19901103, respectively, were mixed with vehicle-controlled cells in the RPMI-1640 medium containing 0.3\% agarose (Beijing Solarbio Science \& Technology Co., Ltd., Beijing, China). These mixtures were seeded on the top of the base layer containing $0.5 \%$ agar in $60 \mathrm{~mm}$ petri dishes (Beijing Solarbio Science \& Technology Co., Ltd.). The cultures were incubated in a $37^{\circ} \mathrm{C}$, humidified, $95 \%$ air and $5 \% \mathrm{CO}_{2}$ atmosphere for three more weeks. The medium was replenished every $72 \mathrm{~h}$. At the end of this assay, only colonies with a diameter $>0.5 \mathrm{~mm}$ was counted by a light microscope (original magnification, x40; Olympus Corporation, Tokyo, Japan).

Flow cytometry analysis of cell cycle arrest. Cell cycle analysis was performed in sub-confluent cells. Following treatment of cells with $20 \mu \mathrm{mol}$ of the three compounds ZINC05463076, ZINC2102846 or ZINC19901103 at for $24 \mathrm{~h}$ at $37^{\circ} \mathrm{C}$, the cells were washed twice with cold PBS and fixed with $70 \%$ ethanol. Next, the cells were washed twice with PBS and $10 \mathrm{mg} / \mathrm{ml}$ RNase A was added. The cells were subsequently stained with propidium iodide (PI; $12.5 \mu \mathrm{g} / \mathrm{ml}$ ) from the Cycle TEST PLUS DNA Reagent kit (BD Pharmingen; BD Biosciences, Franklin Lakes, NJ, USA) and incubated at $4^{\circ} \mathrm{C}$ for $30 \mathrm{~min}$ in the dark. The cells were then subjected to flow cytometry using a FACScan flow cytometer (BD Biosciences). Data acquisition was performed using a flow cytometer (Accuri C6;
BD Biosciences) and cell cycle distribution was determined using FlowJo software (version 10; FlowJo, LLC, Ashland, OR, USA). The number of gated cells in the $\mathrm{G}_{1}, \mathrm{G}_{2} / \mathrm{M}$ or $\mathrm{S}$ cell cycle phase was presented as a percentage. At least 10,000 singlets were analyzed.

Cell extracts and western blot analysis. Cells were grown to approximately $80 \%$ confluence, rinsed twice with cold PBS and lysed in the presence of SDS lysis buffer (Pierce; Thermo Fisher Scientific, Inc.) for $30 \mathrm{~min}$ on ice, according to the manufacturer's instructions. The protein concentration of the lysates was determined using a Pierce BCA Protein Assay kit (Thermo Fisher Scientific, Inc.).

Equal amount of total proteins $(40 \mu \mathrm{g})$ was subjected to $10 \%$ SDS-PAGE and then transferred to a polyvinylidene difluoride membrane (GE Healthcare, Chicago, IL, USA). Membranes were blocked with 5\% skim milk at room temperature for $1 \mathrm{~h}$, followed by incubation with primary antibodies against phosphorylated (p-) ERK1/2 (cat. no. 4370; 1:1,000), ERK1/2 (cat. no. 4060; 1:1,000), p-Akt (cat. no. 4691; 1:1,000), Akt (cat. no. 4691; 1:1,000), Cyclin D1 (cat. no. 2922; 1:1,000), cyclin dependent kinase 2 (CDK2; cat. no. 2546S; 1:1,000), cyclin dependent kinase 4 (CDK4; cat. no. $12790 ; 1: 1,000)$, survivin (cat. no. 8457; 1:10,000) and $\beta$-actin (cat. no. 8457; 1:10,000; all from Cell Signaling Technology, Inc., Danvers, MA, USA) at $4^{\circ} \mathrm{C}$ overnight. Then following three washes with TBST, the membranes were incubated with horseradish peroxidase-conjugated polyclonal goat anti-rabbit IgG secondary antibody (cat. no. sc-2004; 1:10,000; Santa Cruz Biotechnology Inc. Dallas, TX, USA) for $1 \mathrm{~h}$ at room temperature. The membrane was washed with PBS and visualized colorimetrically using 3,3,5,5 tetramethylbenzine solution, which lead to protein quantifying using a gel documentation system (Bio-Rad Laboratories, Inc.). Alternatively, normalization was performed with respect to the amount of b-actin in the same sample. Quantification of band densities was performed using ImageJ software (version 1.6.0_20; National Institutes of Health, Bethesda, MD, USA).

Reverse transcription-quantitative polymerase chain reaction $(R T-q P C R)$. Total RNA was purified from the cell lines, $\mathrm{LNCaP}$, DU-145, PC-3 and LNCaP-AI cells, using QIAGEN RNeasy mini kit (Qiagen, Inc., Valencia, CA, USA) and submitted to DNase treatment (all from Qiagen, Inc.), according to the manufacturer's protocol. cDNAs were generated by reverse transcription from $1 \mu \mathrm{g}$ total RNA. Objective genes were measured by qPCR using SYBR Premix Ex Taq (Takara Bio Inc., Otsu, Japan) and ABI prism 7500 Real-Time PCR System (Applied Biosystems; Thermo Fisher Scientific, Inc.). Primers were synthesized and designed by Shanghai GenePharma Co., Ltd, China. The oligonucleotide primers used were as follows: Extracellular signal-regulated kinase (ERK)1 forward, 5'-GCTGAATCACATCCTGGGTAT-3' and ERK1 reverse, 5'-AGATCTGTATCCTGGCTGGAA-3'; ERK2 forward, 5'-GCAGGTGTTCGACGTGGGAAT-3' and ERK2 reverse, GTGCAGAACATTAGGTGAATA-3'; EGFR forward, 5'-TGT TTGGGACCTCCGGTCAG-3' and EGFR reverse, 5'-GGC AGGTCTTGACGCAGTGG-3'; GAPDH forward, 5'-ATG TTCGTCATGGGTGTGAACCA-3' and GAPDH reverse, 5'-TGGCAGGTTTTTCTAGACGGCAG-3'. Reactions were 

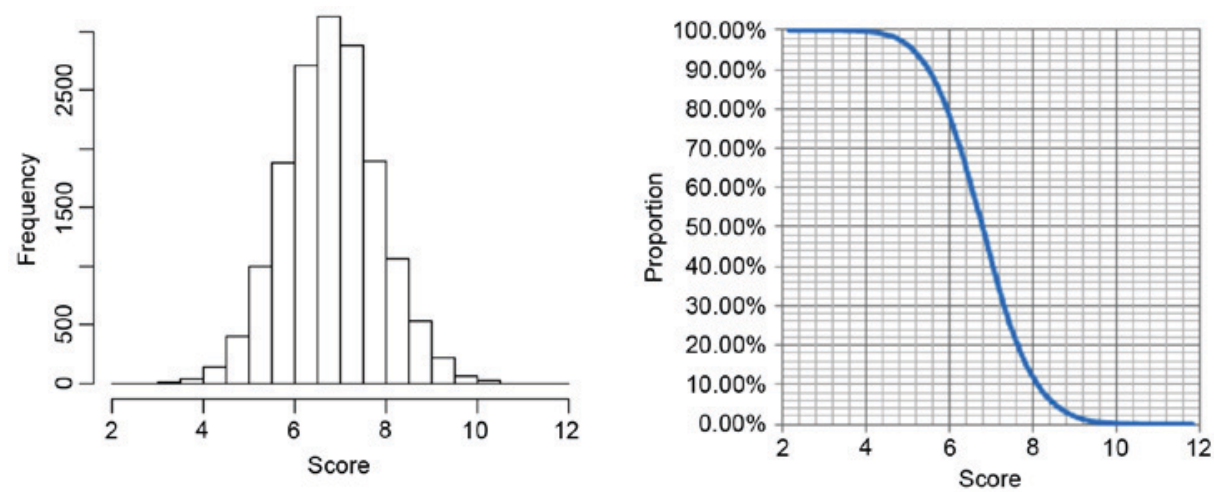

Figure 2. Distribution of docking score of the decoy molecules. Histogram and inverse cumulative distribution of docking scores. Among the 15,966 decoy molecules, only $1 \%$ of molecules have scores $>9.29 \mathrm{pKd}$.

performed for 35 cycles of $95^{\circ} \mathrm{C}$ for $5 \mathrm{~min}, 95^{\circ} \mathrm{C} 30 \mathrm{sec}, 62^{\circ} \mathrm{C}$ for $30 \mathrm{sec}, 30 \mathrm{sec} 72^{\circ} \mathrm{C}$ for $30 \mathrm{sec}$, and a final extension at $72^{\circ} \mathrm{C}$ for $10 \mathrm{~min}$. The quantification cycle $(\mathrm{Cq})$ values obtained for each gene were referenced to GAPDH and quantification was performed using the $2^{-\Delta \Delta C q}$ method (20).

Annexin V-FITC staining. Staining cells with Annexin V-FITC was performed to investigate the cell apoptosis. After harvesting cells and washing with cold PBS, the cells were washed and transferred to a 5-ml culture tube and incubated with $5 \mu \mathrm{l}$ FITC-conjugated Annexin V and $5 \mu \mathrm{l}$ PI for $15 \mathrm{~min}$ at room temperature in the dark (BioLegend, Inc., San Diego, CA, USA). Each sample was added with $400 \mu$ l bind buffer (Gibco; Thermo Fisher Scientific, Inc.) and analyzed by FACS based on CellQuest Research Software (Largo, FL, USA).

Statistical analysis. Assays were repeated in at least three individual experiments. The data are presented as the mean \pm standard deviation. Statistical analyses were determined by one-way analysis of variance using SPSS version 13.0 software (SPSS, Inc., Chicago, IL, USA). P $<0.05$ was considered to indicate a statistically significant difference and $\mathrm{P}<0.01$ was considered to indicate a highly statistically significant difference.

\section{Results}

\section{Molecular docking and synthesis}

Self-docking study. The first step of the presents study was the evaluation of the docking accuracy by a self-docking investigation. Self-docking was performed on the 1 XKK X-ray structure using the Surflex-Dock in GEOM mode. It was revealed that the RMSD between the top pose and co-crystallized poses was 1.689. As shown in Fig. 1, the bind mode of the bound ligand was successfully reproduced. Considering its performance, Surflex-Dock was used for the subsequent virtual screening.

Virtual screening. As shown in Fig. 2, the distribution of the score of EGFR decoy molecules was close to being normal, with a mean of 6.80 and standard deviation of 1.03 . Given that the decoy molecules were selected randomly, the chance that any molecule within the 15,966 is an EGFR kinase ligand are small. As a consequence, the present study took advantage of this distribution to estimate the P-value for a particular
Table I. Three molecules with $\mathrm{P}<0.002$.

\begin{tabular}{lccc}
\hline ZINC identity & Dock score & Crash score & Polar score \\
\hline ZINC05463076 & 11.74 & -1.11 & 2.14 \\
ZINC2102846 & 10.67 & -1.72 & 4.18 \\
ZINC19901103 & 10.59 & -1.75 & 3.52 \\
\hline
\end{tabular}

level of docking score for a given compound. Fig. 2 shows the association between the docking score and the proportion was greater than the corresponding score. In the present study, $99 \%$ of decoy molecules revealed a docking score $<9.29$, which resulted in a P-value of 0.01 . Generally, given any molecule with a docking score of S, a P-value can be determined by accessing the proportion of decoy molecules that have an equal or greater docking score to $\mathrm{S}$. The present study focused on the molecules with $\mathrm{P}<0.01$, which means docking a score $>9.29$. After virtual screening, the top 412 compounds with $\mathrm{P}<0.01$ and molecules with $\mathrm{P}<0.002$ (dock score $\geq 10 \mathrm{pKd}$, crash score $\geq-2 \mathrm{pKd}$ and polar score $\geq 2 \mathrm{pKd}$ ) were selected to be reviewed by eye by a pharmacologist. The pharmacologists in Chongqing Pharmaceutical (Group) Co., Ltd. selected three molecules listed in Table I. ZINC identities of ZINC05463076, ZINC2102846 and ZINC19901103 and their 2-dimensional structures are shown in Fig. 3. The binding modes predicted by docking evaluation for these molecules are shown in Fig. 4.

\section{Biological evaluation}

Cellular anti-proliferative activities. Each synthesized compound, ZINC05463076, ZINC2102846 and ZINC19901103, included in the present study was screened for their cytotoxic and anti-proliferative activity against LNCaP, PC-3 and DU-145 cell lines, as well as the LNCaP-AI cells. An MTT assay was performed to determine the cytotoxicity. The cytotoxic effect of each substance was analyzed at concentrations ranging from 0.01 to $100 \mathrm{mM}$. During this assay, the approved EGFR inhibitor drug gefitinib was used as a reference compound (17). The obtained results are presented in Table II and expressed as $\mathrm{IC}_{50}$ values that reduced the growth of treated cells compared with the untreated controls. 
Table II. MTT assay. $\mathrm{IC}_{50}$ values $(\mu \mathrm{g} / \mathrm{ml})$ for anti-proliferative activity of various extracts towards LNCaP, PC-3, DU-145 and LCNaP-AI cells.

\begin{tabular}{lccrr}
\hline Variable & LNCaP & PC-3 & DU & LNCaP-AI \\
\hline Z76 & $13.34 \pm 1.2$ & $12.65 \pm 1.34$ & $11.67 \pm 1.02$ & $13.19 \pm 0.51$ \\
Z46 & $8.72 \pm 0.91$ & $10.37 \pm 1.27$ & $8.89 \pm 0.27$ & $16.32 \pm 2.31$ \\
Z03 & $11.93 \pm 1.03$ & $11.97 \pm 0.74$ & $12.23 \pm 0.28$ & $14.21 \pm 0.51$ \\
\hline
\end{tabular}

Values are presented as the mean \pm standard error of the mean of three independent Experiments performed in triplicate. Z76, ZINC05463076; Z46, ZINC2102846; Z03, ZINC19901103; IC $_{50}$, half-maximal inhibitory concentration.

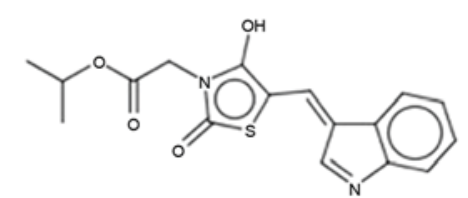

ZINC05463076

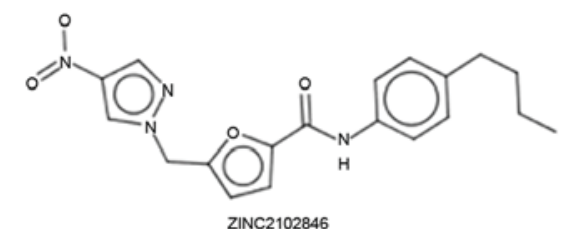

ZINC2102846

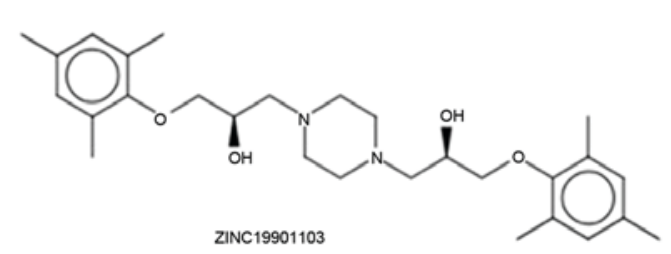

ZINC19901103

Figure 3. Two-dimensional structures of molecules of interest.

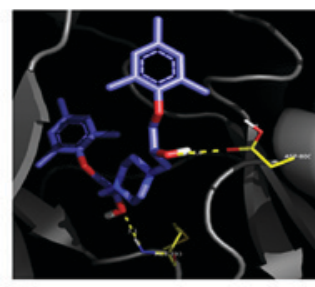

ZINC19901003

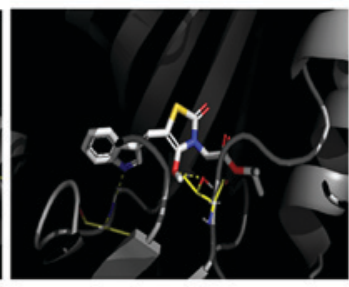

ZINC05463076

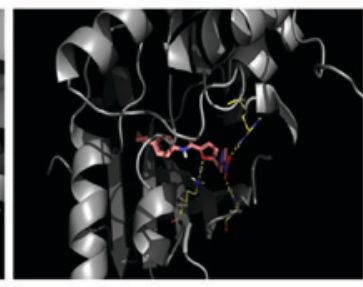

ZINC02102846

Figure 4. The binding modes predicted by docking calculation.

As presented in Table II, the $\mathrm{IC}_{50}$ values of ZINC05463076 in the LNCaP, PC-3, DU and LNCaP-AI cells were $13.34 \pm 1.2$, $12.65 \pm 1.34,11.67 \pm 1.02$ and $13.19 \pm 0.51 \mu \mathrm{mol} / 1$, respectively. The inhibitory effects of ZINC05463076 gradually increased as the concentration increased, achieving a maximal inhibition of $85 \%$ ( $\mathrm{LNCaP}$ ) and almost $76 \%$ in other cells at the maximum concentration. In LNCaP, PC-3, DU and LNCaP-AI cells, ZINC05463076 exhibited a maximum inhibition rate of almost $70 \%$ in all the cell lines, respectively, with $\mathrm{IC}_{50}$ values of $8.72 \pm 0.91,10.37 \pm 1.27,8.89 \pm 0.27$ and $16.32 \pm 2.31 \mu \mathrm{mol} / 1$, respectively. The $\mathrm{IC}_{50}$ values of ZINC19901103 were $11.93 \pm 1.03,11.97 \pm 0.74,12.23 \pm 0.28$ and $14.21 \pm 0.51 \mu \mathrm{mol} / 1$, in LNCaP, PC-3, DU and LNCaP-AI cells respectively. Additionally, as shown in Fig. 5, the proliferation of LNCaP and LNCaP-AI cell lines were significantly inhibited by treatment with ZINC05463076 in a dose-dependent manner based on colony formation assays (ZINC2102846 and ZINC19901103 exhibited similar effects; data not shown).

Kinase inhibitory activity in vitro. Designed and predicted to be EGFR inhibitors, the activity against tyrosine kinases of the compounds, ZINC05463076, ZINC2102846 and ZINC19901103 were examined by an in vitro kinase assay. The present study determined that all compounds inhibited EGFR activities efficiently, with respective $\mathrm{IC}_{50}$ values. Furthermore,
ELISA analysis quantitatively revealed that treating LNCaP, PC-3, DU-145 and LNCaP-AI cell lines with the compounds decreased the expression level of EGFR significantly (Fig. 6A). Western blotting and RT-qPCR were performed to determine the expression levels of the EGFR family molecules in the cell lines (LNCaP, DU-145, PC-3 and LNCaP-AI cells). mRNA and protein analysis demonstrated that the novel synthetic EGFR inhibitors markedly decreased the expression levels of EGFR and ERK1/2 (Fig. 6B and C; ZINC2102846 and ZINC19901103 revealed similar effects; data not shown). These results demonstrated that the molecules synthesized in the present study maintained kinase inhibitory activity.

Agents induce cell cycle arrest in PCa cells. Flow cytometry was performed to evaluate the capacity of the agents in inhibiting cell cycle progression in a panel of LNCaP, PC-3, DU-145 and LNCaP-AI cells. Compared with gefitinib, treatment with these agents induced $\mathrm{G}_{1}$ cell cycle arrest and accumulation in the $\mathrm{G}_{0} / \mathrm{G}_{1}$ phase, which was identical to the control compound Gefitinib (Fig. 7). To clarify the potential mechanisms underlying these compounds on the cell cycle, the present study investigated the expression levels of main regulators of the $\mathrm{G}_{1} / \mathrm{S}$ transition. Western blot analysis revealed that treatments with these agents significantly decreased the expression levels of CDK1, CDK2 and CDK4, the main cyclins of the $\mathrm{G}_{1}$ cell cycle 

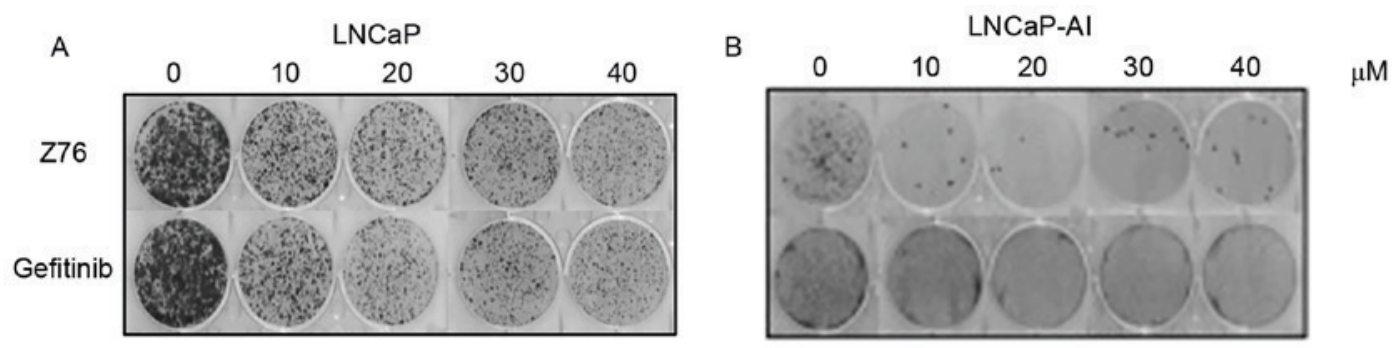

Figure 5. ZINC2102846 inhibited proliferation in (A) LNCaP and (B) LNCaP-AI cells. The cells were treated with increasing doses of the ZINC05463076 or gefitinib and total cell viability was determined by clonogenic assay. Z76, ZINC05463076.
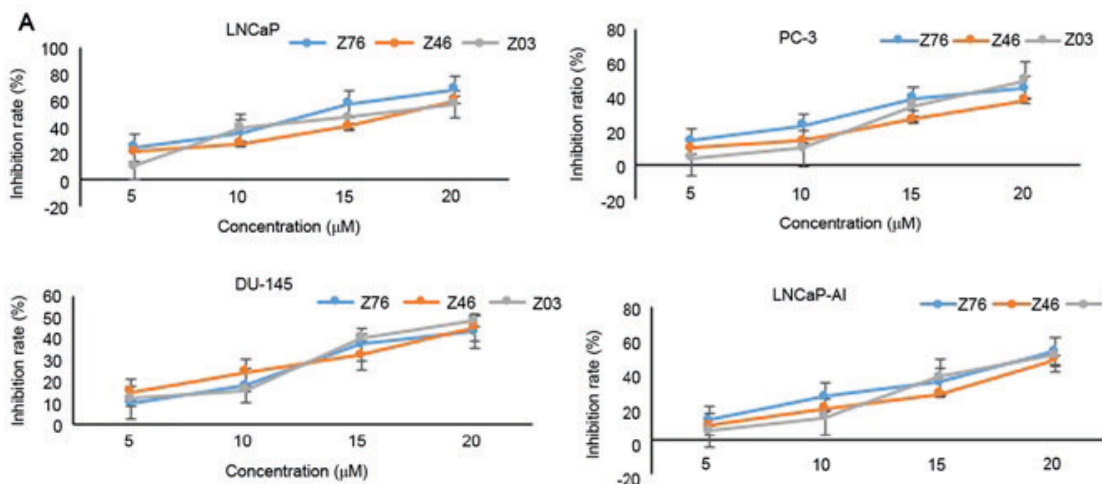

C
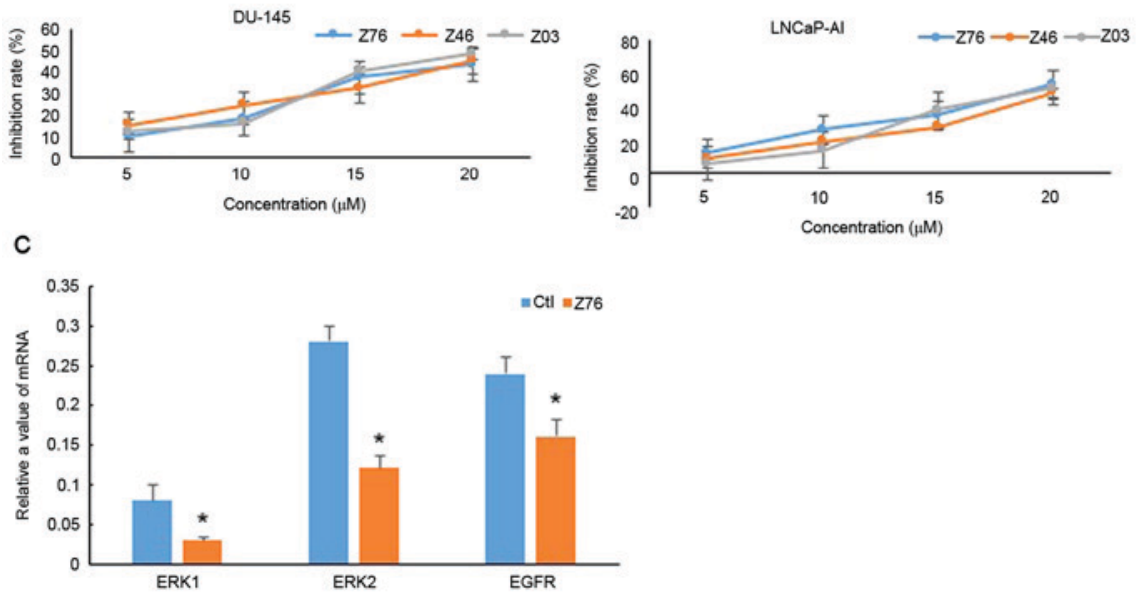

B 276

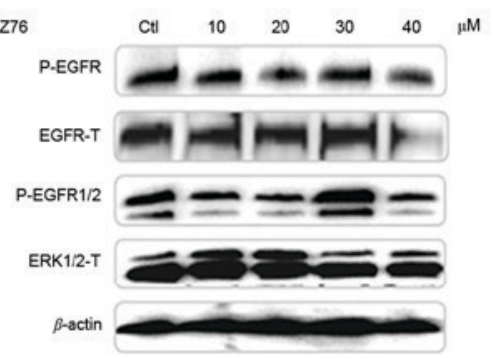

Figure 6. Compounds inhibited EGFR kinase activity. (A) Compounds inhibited EGFR kinase activity in vitro. (B) The kinase profiling assay was analyzed to detect the effects of Z76 on EGFR and ERK1/2 phosphorylation in LNCaP cell lines. (C) Detection of ERK1, ERK2 and EGFR mRNA by reverse transcription-quantitative polymerase chain reaction following treatment of LNCaP cell lines with Z76 (20 $\mu \mathrm{M})$. Z76 vs. the control ("P<0.05). EGFR, epidermal growth factor receptor; ERK, extracellular signal-regulated kinase. Z76, ZINC05463076; Z46, ZINC2102846; or Z03, ZINC19901103.

phase (Fig. 8). The results of the present study suggested that the treatment of PCa cells decreased the expression level of $\mathrm{CDK}$ and regulated subsequent molecules, including survivin, leading to further $\mathrm{G}_{1}$ cell cycle phase arrest.

Agents promote apoptosis in PCa cells. In order to investigate whether the agents of ZINC05463076, ZINC2102846 or ZINC19901103 induced PCa cell apoptosis, the fractions of early (negative) and late (positive) apoptosis in treated cells were determined using a flow cytometer and Annexin V-FITC and PI staining. The results demonstrated that they triggered the significant increase of early and late apoptosis in all the cell lines in a dose-dependent manner. For instance, nearly $40 \%$ of LNCap cells underwent apoptosis treatment, whereas approximately $45 \%$ of the cells experienced apoptosis when the concentration for ZINC05463076 was $20 \mu \mathrm{mol} / \mathrm{l}$. In addition, the present study also performed western blotting to investigate the effects of the compounds in PCa. As is evident in Fig. 8, the agents decreased the expression level of survivin in LNCaP cells lines compared with gefitinib at the same concentration of $50 \mu \mathrm{mol} / 1$, whereas the expression levels of phosphorylated (p)-EGFR and p-ERK1/2 were also decreased (the data for PC-3, DU-145 and LNCaP-AI cells lines are not shown). Thus, the results of the present study revealed that compounds inhibited tumor cell proliferation via cell cycle arrest and apoptosis induction.

\section{Discussion}

EGFR signaling pathway was demonstrated to be highly activated in prostate tumors and leads to significant tumor proliferation and metastasis (18). Inhibiting the EGFR signaling pathway selectively is a potential target for future tumor therapeutic strategies (19). At present, most of the known molecular EGFR-tyrosine kinase inhibitors (TKIs) are anilinoquinazoline chemotypes, including gefitinib and erlotinib. The primary aim of the present study was to investigate for compounds that could substitute existing EGFR-TKIs, particularly gefitinib and expand the scope of drug selections. Thus, the present study rationally synthesized 

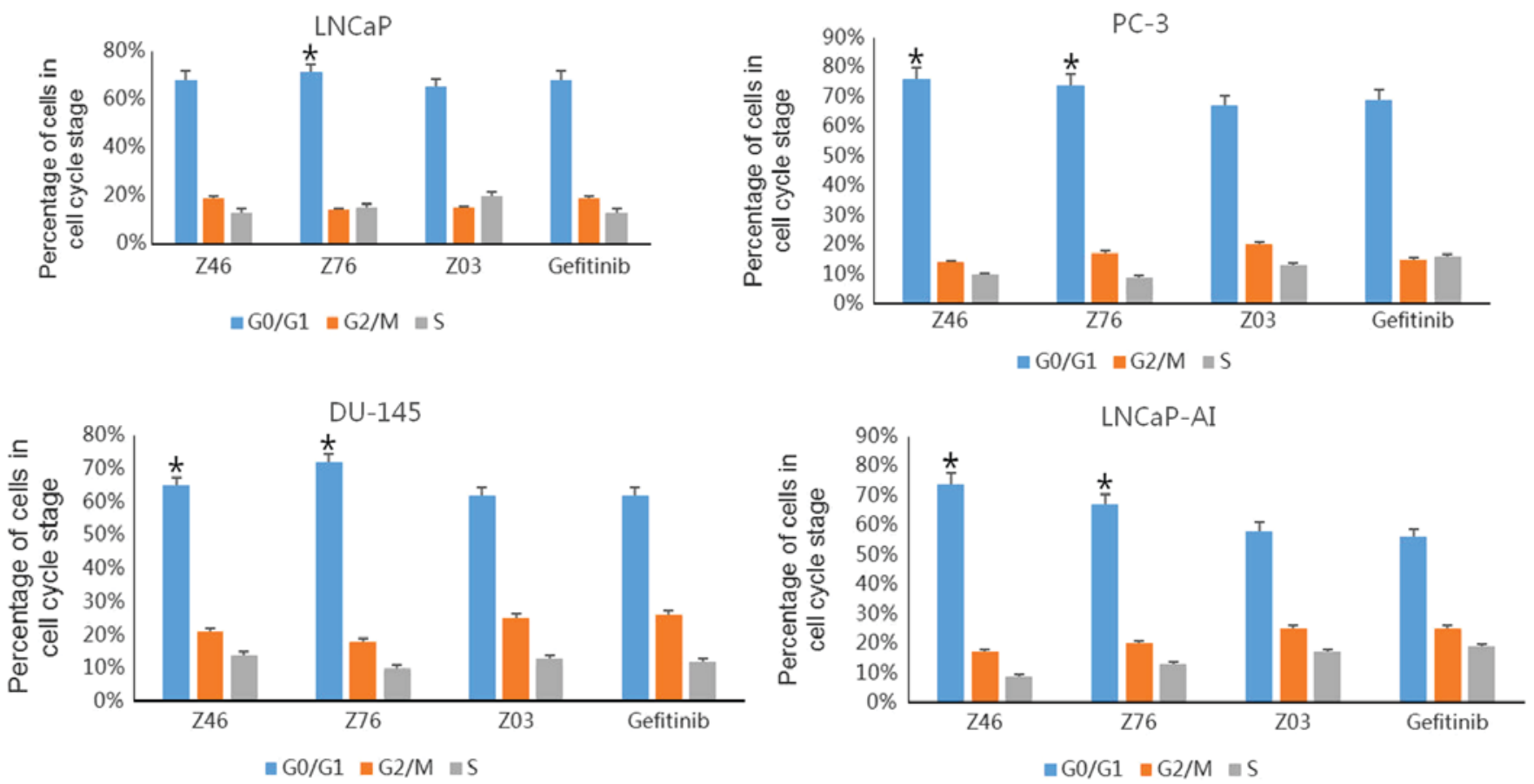

Figure 7. Compounds induced cell cycle arrest in a number of cell lines. LNCaP, DU-145, PC-3 and LNCaP-AI cells were harvested following exposure to ZINC05463076, ZINC2102846 and ZINC19901103 at the indicated concentrations. Cells were fixed for flow cytometry analysis. Z76, Z46 or Z03 vs. the control ("P<0.05). Z76, ZINC05463076; Z46, ZINC2102846; or Z03, ZINC19901103.

A

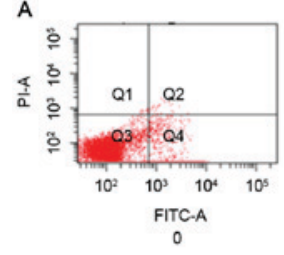

0
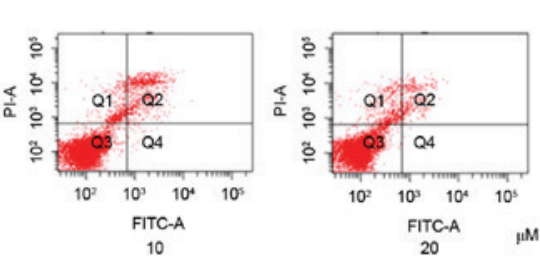

B
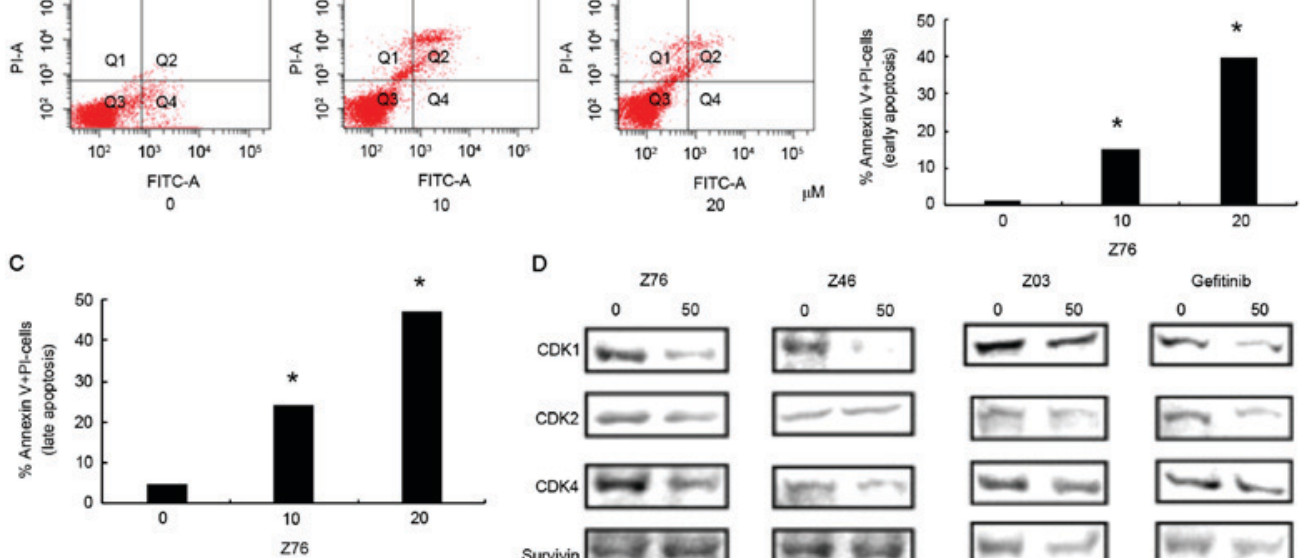

D
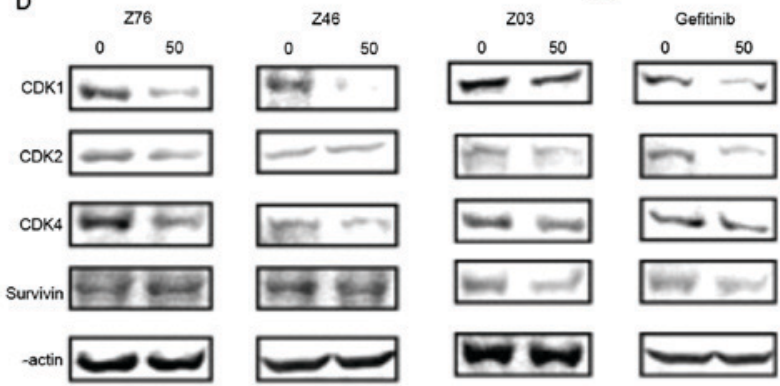

Figure 8. Apoptosis analysis of LNCaP cells in responses to ZINC2102846. Cells were harvested 48 h post-treatment, stained with Annexin V-FITC/PI and then analyzed by (A) flow cytometry. (B) Annexin V positive/PI negative indicated early phase of apoptosis. (C) Annexin V positive/PI positive represented late apoptosis. (D) The proteins associated with apoptosis were increased by treatment with ZINC05463076, ZINC2102846 or ZINC19901103, as revealed by western blotting. "P<0.05 compared with untreated cells. FITC, fluorescein isothiocyanate; PI, propidium iodide; Z76, ZINC05463076; Z46, ZINC2102846; Z03, ZINC19901103; CDK, cyclin-dependent kinase.

a series of derivatives and evaluated their antitumor activities in vitro. ZINC05463076, ZINC2102846 and ZINC19901103 were selected for further investigation due to their distinct antitumor activities in vitro.

A variety of effects have been detected in cancer cells following the treatment of kinase inhibitors, including anti-proliferation, cell cycle arrest and apoptosis (21). The results of the present study demonstrated that these compounds inhibited tumor cell proliferation and suppressed colony formation of the four prostate tumor cell lines markedly, even in the androgen-independent cells. These results demonstrated that the $\mathrm{IC}_{50}$ values of ZINC05463076 in LNCaP, PC-3, DU-145 and LNCaP-AI cell lines were $13.34 \pm 1.2,12.65 \pm 1.34,11.67 \pm 1.02$ and $13.19 \pm 0.51 \mu \mathrm{mol} / 1$, respectively. The compounds of ZINC2102846 and ZINC19901103 revealed similar results to ZINC05463076. The results of the MTT assay demonstrated that the proliferation inhibited in LNCaP cells was weaker 
compared with the other cells. In addition, EGFR inhibitor-mediated colony formation suppression and apoptosis induction in LNCaP cells was more significant compared with that in the other cell lines. Compared with the low expression level of EGFR in other cells, the expression level of EGFR was markedly higher in LNCaP cells, which may account for a higher sensitivity to EGFR inhibitors compared with the others following p-EGFR suppression.

The present study revealed that the agents exerted antitumor effects on tumor cells and promoted apoptosis in a dose-dependent manner. The results from flow cytometry demonstrated that these compounds induced a distinct increase in $G_{0} / G_{1}$ cell cycle arrest and decreased the $G_{2} / M$ and $\mathrm{S}$-phases in various types of cancer cells with concomitant alterations in the mitotic cell cycle-associated proteins CDK1, CDK2 and CDK4. Previous studies demonstrated that the CDK-cyclin-Rb-axis acts as a shifting mechanism in cell progression and cell cycle entry, of which alteration in cell cycle is particularly significant (22-24). The observations of the present study were consistent with a study by Rigas et al (25), which revealed that androgen-independent prostate tumor cells, including LNCaP-AI cells with CDK1/CDK2-inhibitors resulted in cell proliferation inhibition and cell cycle arrest (25). Overexpression of cyclin D1 in LNCaP cell lines may promote S-phase entry and increase colony formation, whereas decreased cyclin D1 induced growth factor-induced cell cycle progression inhibition $(26,27)$. Blocking CDK4/6 selectively significantly may impair the capacity of PCa cells by promoting a robust $\mathrm{G}_{1}$-arrest and induce $\mathrm{PC}$, a xenograft inhibition (28). In addition, tumor cell arrest at checkpoints to repair damage require growth factors to proceed effectively (29). The inhibition of growth factor receptor induced the failure of this process, contributing to cell death (30). The results of the present study revealed that targeting EGFR reduced cell population in $\mathrm{S}$ phase and arrested cells at the $\mathrm{G}_{0} / \mathrm{G}_{1}$ cell cycle phase in prostate cells. Profound tumor cell inhibition was observed; therefore, the cells were sensitized with the compounds of ZINC05463076, ZINC2102846 and ZINC19901103 we synthesized in the present study research.

Molecules, including ERK1/2 are downstream molecules of the EGFR signaling pathway. These molecules have been demonstrated to be constitutively active in most type of cancer (31). The present study revealed that these drugs induced marked reductions in the expression levels of p-ERK1/2 and p-EGFR in a dose-dependent manner, with little effect on the total expression levels of EGFR and ERK in the cell lines by performing western blotting and RT-qPCR. The results indicated that the compounds exerted their effects on p-EGFR protein, decreased the expression level of the upstream EGFR signaling pathway and further resulted in EGFR phosphorylation inhibition. The results of the present study also revealed that the three compounds may inhibit other downstream signaling routes of the EGFR family, including the signal transducer and activator of transcription signaling pathway and phosphoinositide 3-kinase/protein kinase B signaling pathway (32). EGFR was demonstrated to be highly expressed in cancer stem cells, suggesting that targeting EGFR may have an impact on the growth of cancer stem cells and suppress cancer relapse (33). The present study revealed that the inhibition of EGFR and its downstream members affect the viability of prostate tumor cells.

As the smallest member of the IAP family of proteins, survivin was demonstrated to be involved in the development of pancreatic tumors (34). Located in the cytoplasm, mitochondria and nuclei, the protein of survivin is highly expressed in tumor tissues, but undetectable in normal tissues (35). Decreased expression levels of survivin promoted apoptosis by increasing caspase activity and regulating reactive oxygen species production (36). Survivin is expressed in tumor cells and is not present in terminally differentiated cells, thus it is an attractive and promising candidate for chemotherapy and clinical trials (37). The synthesized agents in the present study decreased the expression level of survivin compared with gefitinib (38), which indicated that the compounds may function via EGFR and survivin modulation and subsequently decrease the expression level of survivin. Survivin expression level in the present study also suggested that survivin-mediated apoptosis serves an essential role in the potential mechanism of newly synthesized compounds.

The present study investigated antitumor compounds and their underlying mechanisms of action. It was revealed that the compounds, INC05463076, ZINC2102846 and ZINC19901103, resulted in apoptosis, cell cycle arrest and subsequently inhibition of tumor cell proliferation, including LNCaP, PC-3, DU-145 and LNCaP-AI cells. The compounds demonstrated significant antitumor activities in prostate tumors in vitro. Contribution to survivin-mediated apoptosis and the EGFR signaling pathway blockade may be involved in the mechanisms of these newly synthesized compounds. The present study provided a convenient approach to synthesis and revealed that the three compounds may be promising anticancer agents in $\mathrm{PCa}$ therapy.

\section{Acknowledgements}

The present study was supported by the National Natural Science Foundation of China (grant no. 81302222) and the Natural Science Foundation of Chongqing (grant no. cstc2013jcyjA10141).

\section{Competing interests}

The authors declare that they have no competing interests.

\section{References}

1. Gaudreau PO, Stagg J, Soulières D and Saad F: The present and future of biomarkers in prostate cancer: Proteomics, genomics, and immunology advancements. Biomark Cancer 8 (Suppl 2): S15-S33, 2016.

2. Hong JH and Kim IY: Nonmetastatic castration-resistant prostate cancer. Korean J Urol 55: 153-160, 2014.

3. Wang Y, Kreisberg JI and Ghosh PM: Cross-talk between the androgen receptor and the phosphatidylinositol 3-kinase/Akt pathway in prostate cancer. Curr Cancer Drug Targets 7: 591-604, 2007.

4. Feitelson MA, Arzumanyan A, Kulathinal RJ, Blain SW, Holcombe RF, Mahajna J, Marino M, Martinez-Chantar ML, Nawroth R, Sanchez-Garcia I, et al: Sustained proliferation in cancer: Mechanisms and novel therapeutic targets. Semin Cancer Biol 35 (Suppl): S25-S54, 2015.

5. Kwabi-Addo B, Ozen M and Ittmann M: The role of fibroblast growth factors and their receptors in prostate cancer. Endocr Relat Cancer 11: 709-724, 2004. 
6. Corn PG, Wang F, McKeehan WL and Navone N: Targeting fibroblast growth factor pathways in prostate cancer. Clin Cancer Res 19: 5856-5866, 2013.

7. Chen H, Zhou L, Wu X, Li R, Wen J, Sha J and Wen X: The $\mathrm{PI} 3 \mathrm{~K} / \mathrm{AKT}$ pathway in the pathogenesis of prostate cancer. Front Biosci (Landmark Ed) 21: 1084-1091, 2016.

8. Proverbs-Singh T, Feldman JL, Morris MJ, Autio KA and Traina TA: Targeting the androgen receptor in prostate and breast cancer: Several new agents in development. Endocr Relat Cancer 22: R87-R106, 2015.

9. Cozza G, Pinna LA and Moro S: Protein kinase CK2 inhibitors: A patent review. Expert Opin Ther Pat 22: 1081-1097, 2012.

10. Mahapatra DK, Asati V and Bharti SK: MEK inhibitors in oncology: A patent review (2015-Present). Expert Opin Ther Pat 27: 887-906, 2017

11. Hospital A, Goñi JR, Orozco M and Gelpí JL: Molecular dynamics simulations: Advances and applications. Adv Appl Bioinform Chem 8: 37-47, 2015.

12. Ferreira LG, Dos Santos RN, Oliva G and Andricopulo AD: Molecular docking and structure-based drug design strategies. Molecules 20: 13384-13421, 2015.

13. Rao CM, Yejella RP, Rehman RS and Basha SH: Molecular docking based screening of novel designed chalcone series of compounds for their anti-cancer activity targeting EGFR kinase domain. Bioinformation 11: 322-329, 2015.

14. Li S, Sun X, Zhao H, Tang Y and Lan M: Discovery of novel EGFR tyrosine kinase inhibitors by structure-based virtual screening. Bioorg Med Chem Lett 22: 4004-4009, 2012.

15. Sun XQ, Chen L, Li YZ, Li WH, Liu GX, Tu YQ and Tang Y: Structure-based ensemble-QSAR model: A novel approach to the study of the EGFR tyrosine kinase and its inhibitors. Acta Pharmacol Sin 35: 301-310, 2014.

16. Singh VK and Coumar MS: Ensemble-based virtual screening: Identification of a potential allosteric inhibitor of Bcr-Abl. J Mo Model 23: 218, 2017.

17. Kellenberger E, Rodrigo J, Muller P and Rognan D: Comparative evaluation of eight docking tools for docking and virtual screening accuracy. Proteins 57: 225-242, 2004.

18. Huang N, Shoichet BK and Irwin JJ: Benchmarking sets for molecular docking. J Med Chem 49: 6789-6801, 2006.

19. McNaughton M, Pitman M, Pitson SM, Pyne NJ and Pyne S: Proteasomal degradation of sphingosine kinase 1 and inhibition of dihydroceramide desaturase by the sphingosine kinase inhibitors, SKi or ABC294640, induces growth arrest in androgen-independent LNCaP-AI prostate cancer cells. Oncotarget 7: 16663-16675, 2016.

20. Livak KJ and Schmittgen TD: Analysis of relative gene expression data using real-time quantitative PCR and the 2(-Delta Delta C(T)) method. Methods 25: 402-408, 2001.

21. Lokadasan R, James FV, Narayanan G and Prabhakaran PK: Targeted agents in epithelial ovarian cancer: Review on emerging therapies and future developments. Ecancermedicalscience 10: 626, 2016.

22. Huskey NE, Guo T, Evason KJ, Momcilovic O, Pardo D, Creasman KJ, Judson RL, Blelloch R, Oakes SA, Hebrok M and Goga A: CDK1 inhibition targets the p53-NOXA-MCL1 axis, selectively kills embryonic stem cells, and prevents teratoma formation. Stem Cell Reports 4: 374-389, 2015

23. Chen X, Guo D, Zhu Y, Xian F, Liu S, Wu L and Lou X: Nuclear phosphoproteomics analysis reveals that CDK1/2 are involved in EGF-regulated constitutive pre-mRNA splicing in MDA-MB-468 cells. J Proteomics 141: 77-84, 2016.
24. Xiao X, Wu J, Zhu X, Zhao P, Zhou J, Liu QQ, Zheng L, Zeng M, Liu R and Huang W: Induction of cell cycle arrest and apoptosis in human nasopharyngeal carcinoma cells by ZD6474, an inhibitor of VEGFR tyrosine kinase with additional activity against EGFR tyrosine kinase. Int J Cancer 121: 2095-2104, 2007.

25. Rigas AC, Robson CN and Curtin NJ: Therapeutic potential of CDK inhibitor NU2058 in androgen-independent prostate cancer. Oncogene 26: 7611-7619, 2007.

26. Hurtado A, Pinós T, Barbosa-Desongles A, López-Avilés S, Barquinero J, Petriz J, Santamaria-Martínez A, Morote J, de Torres I, Bellmunt J, et al: Estrogen receptor beta displays cell cycle-dependent expression and regulates the G1 phase through a non-genomic mechanism in prostate carcinoma cells. Cell Oncol 30: 349-365, 2008.

27. Wang H, Zhang C, Rorick A, Wu D, Chiu M, Thomas-Ahner J, Chen Z, Chen H, Clinton SK, Chan KK and Wang Q: CCI-779 inhibits cell-cycle G2-M progression and invasion of castration-resistant prostate cancer via attenuation of UBE2C transcription and mRNA stability. Cancer Res 71: 4866-4876, 2011.

28. Comstock CE, Augello MA, Goodwin JF, de Leeuw R, Schiewer MJ, Ostrander WF Jr, Burkhart RA, McClendon AK, McCue PA, Trabulsi EJ, et al: Targeting cell cycle and hormone receptor pathways in cancer. Oncogene 32: 5481-5491, 2013.

29. Chen J: The cell-cycle arrest and apoptotic functions of p53 in tumor initiation and progression. Cold Spring Harb Perspect Med 6: a026104, 2016.

30. Purev E, Cai D, Miller E, Swoboda R, Mayer T, Klein-Szanto A, Marincola FM, Mick R, Otvos L, Wunner W, et al: Immune responses of breast cancer patients to mutated epidermal growth factor receptor (EGF-RvIII, Delta EGF-R, and de2-7 EGF-R). J Immunol 173: 6472-6480, 2004.

31. Werry TD, Christopoulos A and Sexton PM: Mechanisms of ERK1/2 regulation by seven-transmembrane-domain receptors. Curr Pharm Des 12: 1683-1702, 2006.

32. Uribe P and Gonzalez S: Epidermal growth factor receptor (EGFR) and squamous cell carcinoma of the skin: Molecular bases for EGFR-targeted therapy. Pathol Res Pract 207: 337-342, 2011.

33. Karandish F and Mallik S: Biomarkers and targeted therapy in pancreatic cancer. Biomark Cancer 8 (Suppl 1): S27-S35, 2016.

34. Rivadeneira DB, Caino MC, Seo JH, Angelin A, Wallace DC, Languino LR and Altieri DC: Survivin promotes oxidative phosphorylation, subcellular mitochondrial repositioning, and tumor cell invasion. Sci Signal 8: ra80, 2015.

35. Chen X, Duan N, Zhang C and Zhang W: Survivin and tumorigenesis: Molecular mechanisms and therapeutic strategies. J Cancer 7: 314-323, 2016.

36. Lim EJ, Heo J and Kim YH: Tunicamycin promotes apoptosis in leukemia cells through ROS generation and downregulation of survivin expression. Apoptosis 20: 1087-1098, 2015.

37. Garg H, Suri P, Gupta JC, Talwar GP and Dubey S: Survivin: A unique target for tumor therapy. Cancer Cell Int 16: 49, 2016.

38. Hakonen E, Ustinov J, Palgi J, Miettinen PJ and Otonkoski T: EGFR signaling promotes $\beta$-cell proliferation and survivin expression during pregnancy. PLoS One 9: e93651, 2014.

This work is licensed under a Creative Commons Attribution-NonCommercial-NoDerivatives 4.0 International (CC BY-NC-ND 4.0) License. 